\title{
A PAIR OF ARBITRARILY-ORIENTED COPLANAR CRACKS IN AN ANISOTROPIC ELASTIC SLAB
}

\author{
W. T. $A N G^{1}$ \\ (Received 23 August 1989; revised 15 May 1990)

\begin{abstract}
The problem of an anisotropic elastic slab containing two arbitrarily-oriented coplanar cracks in its interior is considered. Using a Fourier integral transform technique, we reduce the problem to a system of simultaneous finite-part singular integral equations which can be solved numerically. Once the integral equations are solved, relevant quantities such as the crack energy can be readily computed. Numerical results for specific examples are obtained.
\end{abstract}

\section{Introduction}

Anisotropic materials have numerous applications in modern technology. Fibre-reinforced composites which are widely used in engineering can be reasonably modelled as anisotropic and inextensible along the fibre direction (see, e.g., Spencer [15]). The determination of elastic displacements and stresses in cracked anisotropic materials is, therefore, a subject of considerable practical importance.

The static displacements and stresses in an infinite anisotropic elastic medium containing a single planar crack were obtained by Stroh [16]. From a practical point of view, the usefulness of these displacements and stresses is necessarily restricted to situations where the crack interacts negligibly with the outer boundary of the material. Clements [5] and, more recently, Ang $[2,3]$ examined the interaction of the crack with the boundary by placing the crack in an infinitely long anisotropic elastic slab. The plane containing the crack is perpendicular and parallel to the boundary of the slab in [2] and [5], respectively, while in [3] it is arbitrarily inclined to the boundary.

\footnotetext{
${ }^{1}$ Engineering Mathematics Section, Universiti Sains Malaysia, Perak, Malaysia.

(C) Copyright Australian Mathematical Society 1991, Serial-fee code 0334-2700/91
} 
Other related work on cracked anisotropic slabs may also be found in Ang [4], Clements and Tauchert [6], and Hill and Clements [9].

The problem of two or more coplanar cracks in an infinite transverselyisotropic material was examined by Konishi [12] and Krenk [13]. Dhaliwal [7] studied the interaction of two coplanar cracks in an infinitely-long orthotropic slab, with the cracks being parallel to the boundary of the slab.

The present paper considers the problem of two coplanar cracks lying on an arbitrary plane in the interior of a general anisotropic elastic slab. Through the use of a Fourier integral transform technique, the problem is reduced to a system of simultaneous finite-part singular integral equations, which can be solved numerically by using collocation methods described by Loakimidis [10] and Kaya and Erdogan [11]. Once the integral equations are solved, quantities of interest such as the crack energy can be calculated readily. $\mathrm{Nu}$ merical results for specific cases of the problem are obtained by solving these integral equations.

\section{Statement of the problem}

Referred to an $O x_{1} x_{2} x_{3}$ Cartesian coordinate system, consider an anisotropic elastic material which occupies the region between the planes $x_{2}=h$ and $x_{2}=-h$, where $h$ is a given real positive number. In its interior, the slab contains two coplanar cracks $a<\left|x_{1} \sin \theta-x_{2} \cos \theta\right|<b, x_{1} \cos \theta+x_{2} \sin \theta=$ 0 , for all $x_{3}$, where the angle $\theta$ lies between $0^{\circ}$ and $360^{\circ}$, and $a$ and $b$ are real positive numbers with $b|\cos \theta|<h$. That is, a normal vector to the plane containing the cracks is given by $[\cos \theta, \sin \theta, 0]$ (see Figure 1). The planes $x_{2}=h$ and $x_{2}=-h$ are free of tractions, and the cracks are opened up by internal stresses which are independent of the coordinate $x_{3}$. The problem is to determine the displacements and the stresses throughout the slab.

\section{Equations of anisotropic elasticity}

The equilibrium equations for anisotropic elasticity are given by the system of partial differential equations

$$
c_{i j k p} \frac{\partial^{2} u_{k}}{\partial x_{j} \partial x_{p}}=0,
$$

where the Latin subscripts take the values of 1,2 and 3, $x_{k}$ are the spatial coordinates with respect to a Cartesian coordinate frame, $u_{k}$ are the Cartesian 


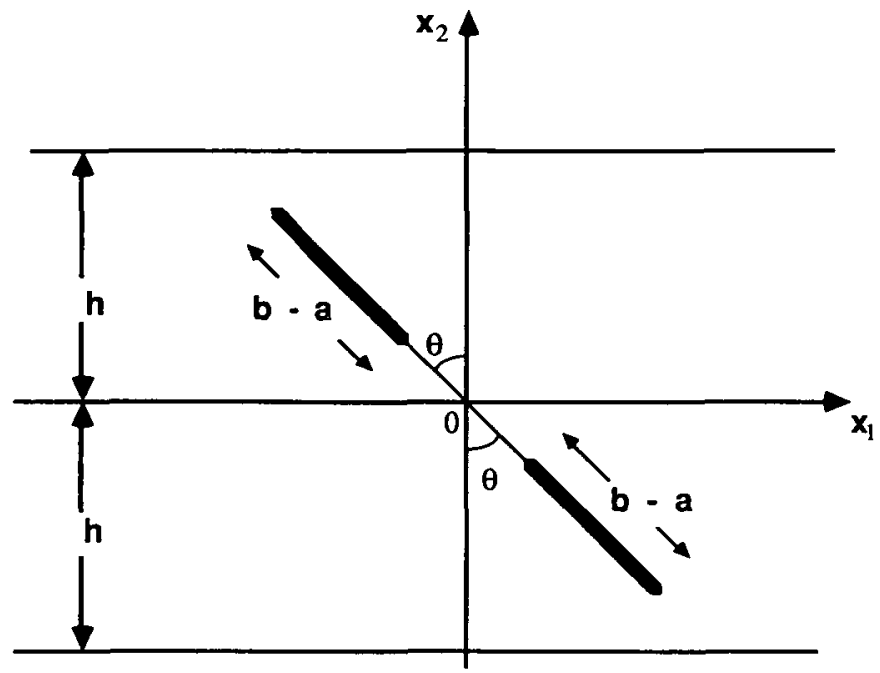

FIGURE 1. A pair of arbitrarily-oriented coplanar cracks in an anisotropic slab.

displacements, and $c_{i j k p}$ are the elastic moduli of the material. The elastic moduli $c_{i j k p}$ satisfy the symmetry conditions

$$
c_{i j k p}=c_{j i k p}=c_{i j p k}=c_{k p i j} \text {, }
$$

and the equality

$$
c_{i j k p} h_{i j} h_{k p}>0
$$

where $h_{i j}(i, j=1,2,3)$ are any arbitrary real numbers, not all of which are zero. The usual convention of summing over a repeated index is assumed here only for Latin subscripts.

If the displacements $u_{k}$ are free of the coordinate $x_{3}$, then (3.1) admits solutions of the form (Ang [3])

$$
u_{k}=\operatorname{Re}\left[\sum_{\alpha} A_{k \alpha}(\rho) f_{\alpha}\left(z_{\alpha}(\rho)\right)\right],
$$

where $\operatorname{Re}$ denotes the real part of a complex number, the sum over $\alpha$ is from 1 to $3, f_{\alpha}\left(z_{\alpha}\right)$ are differentiable functions of $z_{\alpha}, z_{\alpha}=\left(a_{j 1}+\tau_{\alpha} a_{j 2}\right) x_{j}$, $\rho$ is a real parameter, $a_{i j}=a_{i j}(\rho)$ for our purpose here are taken to be

$$
\left[a_{i j}(\rho)\right]=\left[\begin{array}{lcl}
\sin \rho & \cos \rho & 0 \\
-\cos \rho & \sin \rho & 0 \\
0 & 0 & 1
\end{array}\right],
$$

$\tau_{\alpha}=\tau_{\alpha}(\rho)$ are the solutions with positive imaginary parts of the characteristic equation,

$$
\operatorname{det}\left[c_{i 1 k 1}+\sigma(\tau)\left\{c_{i 1 k 2}+c_{i 2 k 1}\right\}+\sigma^{2}(\tau) c_{i 2 k 2}\right]=0,
$$


where $\sigma(\tau)=\left(a_{21}+\tau a_{22}\right) /\left(a_{11}+\tau a_{12}\right)$, and $A_{k \alpha}=A_{k \alpha}(\rho)$ are the nonzero solutions of the system

$$
\left[c_{i 1 k 1}+\sigma\left(\tau_{\alpha}\right)\left\{c_{i 1 k 2}+c_{i 2 k 1}\right\}+\sigma^{2}\left(\tau_{\alpha}\right) c_{i 2 k 2}\right] A_{k \alpha}=0 .
$$

Note that the inequality in (3.3) is violated if (3.6) admits a real solution. Hence, the solutions of (3.6) are complex and occur in conjugate pairs. It is assumed here that they are distinct.

Using the generalised Hooke's law

$$
\sigma_{l k}=c_{l k m p} \frac{\partial u_{m}}{\partial x_{p}}
$$

we find that the Cartesian stresses $\sigma_{i j}$ corresponding to the displacements in (3.4) are given by

$$
\sigma_{k p}=\operatorname{Re}\left[\sum_{\alpha} L_{k p \alpha}(\rho) f_{\alpha}^{\prime}\left(z_{\alpha}(\rho)\right)\right],
$$

where the prime denotes differentiation with respect to $z_{\alpha}$ and $L_{i j \alpha}=$ $\left(a_{p 1}+\tau_{\alpha} a_{p 2}\right) c_{i j k p} A_{k \alpha}$.

\section{Solution of the problem}

Our mathematical objective is to solve (3.1) subject to the conditions

$$
\sigma_{k 2}\left(x_{1}, h\right)=\sigma_{k 2}\left(x_{1},-h\right)=0 \text { for }-\infty<x_{1}<\infty,
$$

and

$$
a_{p 2}(\theta) \sigma_{k p} \rightarrow-P_{k}\left(a_{p 1}(\theta) x_{p}\right) \quad \text { as } a_{p 2}(\theta) x_{p} \rightarrow 0 \text { for } a<\left|a_{p 1}(\theta) x_{p}\right|<b,
$$

where $P_{k}(x)$ are suitably prescribed functions of $x$. For our discussion here, we assume that the $P_{k}(x)$ are even functions of $x$.

Let the displacements and the stresses be respectively given by

$$
\begin{aligned}
u_{k}=\operatorname{Re} \int_{0}^{\infty} \sum_{\alpha}\left\{2 A_{k \alpha}(\phi) E_{\alpha}(\xi) \sinh \left(i \xi z_{\alpha}(\phi)\right)\right. \\
+H\left(a_{p 2}(\theta) x_{p}\right) A_{k \alpha}(\theta) G_{\alpha}^{+}(\xi) \exp \left(i \xi z_{\alpha}(\theta)\right) \\
\left.+H\left(-a_{p 2}(\theta) x_{p}\right) A_{k \alpha}(\theta) G_{\alpha}^{-}(\xi) \exp \left(-i \xi z_{\alpha}(\theta)\right)\right\} d \xi,
\end{aligned}
$$

and

$$
\begin{aligned}
\sigma_{k m}=\operatorname{Re} \int_{0}^{\infty} \sum_{\alpha}\left\{2 L_{k m \alpha}(\phi) E_{\alpha}(\xi) \cosh \left(i \xi z_{\alpha}(\phi)\right)\right. \\
+H\left(a_{p 2}(\theta) x_{p}\right) L_{k m \alpha}(\theta) G_{\alpha}^{+}(\xi) \exp \left(i \xi z_{\alpha}(\theta)\right) \\
\left.-H\left(-a_{p 2}(\theta) x_{p}\right) L_{k m \alpha}(\theta) G_{\alpha}^{-}(\xi) \exp \left(-i \xi z_{\alpha}(\theta)\right)\right\} i \xi d \xi
\end{aligned}
$$


where $\phi=\pi / 2, i=(-1)^{1 / 2}, H(x)$ is the Heaviside unit-step function and $E_{\alpha}(\xi), G_{\alpha}^{+}(\xi)$ and $G_{\alpha}^{-}(\xi)$ are arbitrary functions yet to be determined.

Our choice of $u_{k}$ and $\sigma_{k j}$ in (4.3) and (4.4) requires us to impose the continuity conditions

$$
\left[a_{p 2}(\theta) \sigma_{k p}\right]^{+}-\left[a_{p 2}(\theta) \sigma_{k p}\right]^{-}=0 \text { for }\left|a_{p 1}(\theta) x_{p}\right| \geq 0,
$$

and

$$
\left[u_{k}\right]^{+}-\left[u_{k}\right]^{-}=0 \text { for } 0<\left|a_{p 1}(\theta) x_{p}\right|<a, b<\left|a_{p 1}(\theta) x_{p}\right|<\infty,
$$

where $\left[f\left(x_{1}, x_{2}\right)\right]^{+}$and $\left[f\left(x_{1}, x_{2}\right)\right]^{-}$denote the limiting values of $f\left(x_{1}, x_{2}\right)$ as $a_{j 2}(\theta) x$, approaches 0 from above and below respectively.

From (4.4), it is easy to verify that conditions (4.5) hold if we choose

$$
G_{\alpha}^{+}(\xi)=M_{\alpha p}(\theta) \psi_{p}(\xi) \text { and } G_{\alpha}^{-}(\xi)=M_{\alpha p}(\theta) \bar{\psi}_{p}(\xi),
$$

where the bar denotes the complex conjugate of a complex number, $\psi_{p}$ are arbitrary functions yet to be determined and $\left[M_{\alpha p}(\theta)\right]$ is the inverse of $\left[a_{j 2}(\theta) L_{k j \alpha}(\theta)\right]$.

Using (4.3) and (4.7), we find that

$$
\begin{aligned}
{\left[u_{k}\right]^{+}-\left[u_{k}\right]^{-}=\operatorname{Re} \int_{0}^{\infty} } & \sum_{\alpha}\left\{A_{k \alpha}(\theta) M_{\alpha p}(\theta)-\bar{A}_{k \alpha}(\theta) \bar{M}_{\alpha p}(\theta)\right\} \\
& \times \psi_{p}(\xi) \exp \left(i \xi a_{q 1}(\theta) x_{q}\right) d \xi .
\end{aligned}
$$

Since $P_{k}(x)$ are assumed to be even functions of $x$, in order to satisfy conditions (4.6), it is sufficient to let

$$
\psi_{p}(\xi)=i \int_{a}^{b} r_{p}(t) \cos (\xi t) d t
$$

where $r_{p}(t)$ are real arbitrary functions to be found. Then, from (4.8), we obtain

$$
\begin{array}{r}
{\left[u_{k}\right]^{+}-\left[u_{k}\right]^{-}=i \frac{\pi}{2} \sum_{\alpha}\left\{A_{k \alpha}(\theta) M_{\alpha p}(\theta)-\bar{A}_{k \alpha}(\theta) \bar{M}_{\alpha p}(\theta)\right\} r_{p}\left(a_{q 1}(\theta) x_{q}\right)} \\
\text { for } a<a_{p 1}(\theta) x_{p}<b .
\end{array}
$$

By using a Fourier inversion theorem which is given in Sneddon [14], we find that conditions (4.1) are satisfied if and only if

$$
\int_{-\infty}^{\infty} \sigma_{k 2}(\xi, h) \exp (-i \lambda \xi) d \xi=\int_{-\infty}^{\infty} \sigma_{k 2}(\xi,-h) \exp (-i \lambda \xi) d \xi=0
$$

where $\lambda$ is some real constant. 
Using the result (Gradshtein and Ryzhik [8])

$$
\begin{aligned}
& \int_{0}^{\infty} \frac{x \sin (a x) \cos (b x) d x}{x^{2}+\beta^{2}}=\frac{\pi}{2} \exp (-a \beta) \cosh (b \beta) \\
& \qquad \text { for } 0<b<a \text { and } \operatorname{Re} \beta>0,
\end{aligned}
$$

we find that, from (4.4), (4.7) and (4.9), conditions (4.11) can be written as (for $\lambda>0$ )

$$
\begin{aligned}
\sum_{\alpha}\left\{L_{2 k \alpha}(\phi) E_{\alpha}(\lambda) \exp \left[i \lambda \tau_{\alpha}(\phi) h\right]-\bar{L}_{k 2 \alpha}(\phi) \bar{E}_{\alpha}(\lambda) \exp \left[i \lambda \bar{\tau}_{\alpha}(\phi) h\right]\right\} \\
=-i \sum_{\alpha} D_{\alpha}^{2}(\theta) L_{2 k \alpha}(\theta) M_{\alpha p}(\theta) \exp \left[i \lambda h D_{\alpha}(\theta)\left(-a_{12}(\theta)+\tau_{\alpha}(\theta) a_{11}(\theta)\right)\right] \\
\quad \times \int_{a}^{b} r_{p}(t) \cosh \left[i \lambda t D_{\alpha}(\theta)\right] d t
\end{aligned}
$$

where $D_{\alpha}(\theta)=\left[a_{11}(\theta)+\tau_{\alpha}(\theta) a_{12}(\theta)\right]^{-1}$.

Equations (4.13) (together with their complex conjugates) can now be solved for $E_{\alpha}(\lambda)$. We obtain (for $\lambda>0$ )

$$
E_{\alpha}(\lambda)=\exp \left[i \lambda h \tau_{\alpha}(\phi)\right] \int_{a}^{b} Y_{\alpha k}(\lambda) K_{k p}(\lambda, t) r_{p}(t) d t
$$

where $\left[Y_{\alpha k}(\lambda)\right]$ is the inverse of $\left[Z_{\alpha k}(\lambda)\right]$ and

$$
\begin{gathered}
Z_{k \beta}(\lambda) \\
=\sum_{\alpha}\left\{\bar{L}_{k 2 \alpha}(\phi) \bar{M}_{\alpha q}(\phi) L_{q 2 \beta}(\phi) \exp \left[2 i \lambda h\left(\tau_{\beta}(\phi)-\bar{\tau}_{\alpha}(\phi)\right)\right]-\delta_{\alpha \beta} L_{k 2 \beta}(\phi)\right\} \\
K_{k p}(\lambda, t)=i \sum_{\alpha} \sum_{\beta}\left\{\delta_{\alpha \beta} \bar{D}_{\beta}^{2}(\theta) \bar{L}_{k 2 \beta}(\theta) \bar{M}_{\beta p}(\theta) \cosh \left[i \lambda t \bar{D}_{\beta}(\theta)\right]\right. \\
\times \exp \left[-i \lambda h \bar{D}_{\beta}(\theta)\left(-a_{12}(\theta)+\bar{\tau}_{\beta}(\theta) a_{11}(\theta)\right)\right] \\
-\bar{M}_{\alpha q}(\phi) D_{\beta}^{2}(\theta) \bar{L}_{k 2 \alpha}(\phi) L_{q 2 \beta}(\theta) M_{\beta p}(\theta) \cosh \left[i \lambda t D_{\beta}(\theta)\right] \\
\left.\times \exp \left[i \lambda h\left\{D_{\beta}(\theta)\left(-a_{12}(\theta)+\tau_{\beta}(\theta) a_{11}(\theta)\right)-2 \bar{\tau}_{\alpha}(\phi)\right\}\right]\right\}, \quad(4.15)
\end{gathered}
$$

where $\delta_{\alpha \beta}$ denotes the Kronecker-delta.

From (4.4), (4.7), (4.9) and (4.14) together with the limit (which can be worked out using a results in Gradshtein and Ryzhik [8])

$$
\lim _{y \rightarrow 0^{+}} \int_{0}^{\infty} 2 \xi \exp (-\xi y) \cos (\xi t) \cos (\xi u) d \xi=-(t-u)^{-2}-(t+u)^{-2}
$$


conditions (4.2) give rise to the system of simultaneous finite-part singular integral equations

$f_{a}^{b} \frac{r_{k}(t) d t}{(t-u)^{2}}+\int_{a}^{b}\left(\frac{r_{k}(t)}{(t+u)^{2}}+\Omega_{k s}(t, u) r_{s}(t)\right) d t=-2 P_{k}(u)$ for $a<u<b$,

where $f$ denotes that the integral is to be interpreted in Hadamard finite-part sense and

$$
\begin{aligned}
\Omega_{k s}(t, u)=4 \int_{0}^{\infty} & \operatorname{Re} \sum_{\alpha} L_{k p \alpha}(\phi) a_{p 2}(\theta) Y_{\alpha q}(\xi) K_{q s}(\xi, t) \\
& \times \exp \left[i \xi h \tau_{\alpha}(\phi)\right] \cosh \left(i \xi\left[a_{11}(\theta)+a_{21}(\theta) \tau_{\alpha}(\phi)\right] u\right) i \xi d \xi .
\end{aligned}
$$

Equations (4.17) can be solved numerically by employing the collocation techniques described in [10] and [11]. We make the approximation

$$
r_{k}(t) \approx \sqrt{1-\left(\frac{t-a-d}{d}\right)^{2}} \sum_{n=1}^{N} \alpha_{k}^{n} U_{n-1}\left(\frac{t-a-d}{d}\right) \text { for } a<t<b,
$$

where $2 d=b-a, U_{n}(x)$ is the $n$th order Chebyshev polynomial of the second kind and $\alpha_{k}^{n}(k=1,2,3 ; n=1,2, \ldots, N)$ are constant coefficients to be determined.

Substituting (4.19) into (4.17), we obtain for $-1<s<1$

$$
\sum_{n=1}^{N} \frac{\alpha_{q}^{n}}{d}\left\{\left[-\pi n U_{n-1}(s)+F^{n}(s)\right] \delta_{k q}+J_{k q}^{n}(s)\right\}=-2 P_{k}(d s+a+d),
$$

where

$$
\begin{gathered}
F^{n}(s)=\int_{-1}^{1} \frac{\sqrt{1-r^{2}} U_{n-1}(r) d r}{\left(r+s+2+2 a d^{-1}\right)^{2}} \\
J_{k q}^{n}(s)=\int_{-1}^{1} d^{2} \sqrt{1-r^{2}} U_{n-1}(r) \Omega_{k q}(r d+a+d, s d+a+d) d r .
\end{gathered}
$$

The integrals in (4.21) can be accurately calculated using the numerical quadrature (25.4.40) in Abramowitz and Stegun [1].

Choosing $s$ in (4.20) to be given by

$$
s=s_{p}=\cos (\{2 p-1\} \pi /(2 N)) \text { for } p=1,2, \ldots, N,
$$


we find that equations (4.20) give rise to a system of $3 N$ linear algebraic equations in ( $3 N$ unknowns) $\alpha_{k}^{n}$. The constants $\alpha_{k}^{n}$ can be determined by solving these equations.

\section{Calculation of crack energy}

The crack energy $U$ for the crack $a<x_{1} \sin \theta-x_{2} \cos \theta<b, x_{1} \cos \theta+$ $x_{2} \sin \theta=0$, is defined by the integral

$$
U=\frac{1}{2} \int_{a}^{b} P_{k}(\phi) \Delta u_{k}(\phi) d \phi,
$$

where $P_{k}(\phi)$ give the traction distribution over the crack surface and $\Delta u_{k}(\phi)$ are the differences between the displacements $u_{k}$ on the top and the bottom faces of the crack as given in (4.10). On a practical note, the crack energy is an important quantity for examining the stability of the crack.

The use of (4.10), (4.19) and (5.1) yields

$$
\begin{aligned}
U \approx & \frac{\pi}{4} \sum_{\beta}\left\{A_{k \beta}(\theta) M_{\beta p}(\theta)-\bar{A}_{k \beta}(\theta) \bar{M}_{\beta p}(\theta)\right\} i d \\
& \times \sum_{n=1}^{N} \alpha_{p}^{n} \int_{-1}^{1} \sqrt{1-r^{2}} P_{k}(r d+a+d) U_{n-1}(r) d r .
\end{aligned}
$$

For the special case where $P_{k}(x)=T_{k}$, where $T_{k}$ are constants, using the orthogonality relation for Chebyshev polynomials, (5.2) becomes

$$
U \approx \frac{\pi^{2}}{8} \sum_{\beta}\left\{A_{k \beta}(\theta) M_{\beta p}(\theta)-\bar{A}_{k \beta}(\theta) \bar{M}_{\beta p}(\theta)\right\} i d \alpha_{p}^{1} T_{k} .
$$

\section{Specific examples}

In this section, the analysis in Section 4 is applied to solve a problem involving a particular transversely-isotropic slab which contains two coplanar cracks.

The elastic behaviour of a transversely-isotropic material is characterised by five independent constants $A, N, F, C$ and $L$. If the transverse planes of the material are parallel to the $O x_{2} x_{3}$ plane, the only nonzero $c_{i j k p}$ are related to these five constants by

$$
\begin{gathered}
A=c_{2222}, \quad C=c_{1111}, \quad F=c_{1122}=c_{2211}=c_{1133}=c_{3311}, \\
(A-N) / 2=c_{2323}=c_{3232}=c_{3223}=c_{2332}, \\
L=c_{1212}=c_{2121}=c_{2112}=c_{1313}=c_{3131}=c_{3113} .
\end{gathered}
$$


Consequently, the system (3.1) reduces to

$$
\begin{gathered}
C \frac{\partial^{2} u_{1}}{\partial x_{1}^{2}}+L \frac{\partial^{2} u_{1}}{\partial x_{2}^{2}}+(F+L) \frac{\partial^{2} u_{2}}{\partial x_{1} \partial x_{2}}=0 \\
A \frac{\partial^{2} u_{2}}{\partial x_{2}^{2}}+L \frac{\partial^{2} u_{2}}{\partial x_{1}^{2}}+(F+L) \frac{\partial^{2} u_{1}}{\partial x_{1} \partial x_{2}}=0 \\
L \frac{\partial^{2} u_{3}}{\partial x_{1}^{2}}+\frac{1}{2}(A-N) \frac{\partial^{2} u_{3}}{\partial x_{2}^{2}}=0
\end{gathered}
$$

and the characteristic equation (3.6) becomes

$$
\left[\frac{1}{2}(A-N) \sigma^{2}(\tau)+L\right]\left[A L \sigma^{4}(\tau)-\left(F^{2}+2 F L-A C\right) \sigma^{2}(\tau)+C L\right]=0 .
$$

If we take

$$
\sigma^{2}\left(\tau_{3}\right)=2 L /(N-A),
$$

then $\tau_{1}$ and $\tau_{2}$ can be obtained from the roots of the quartic factor in $\sigma(\tau)$ in (6.3). From (3.7), we have

$$
\left[A_{k \alpha}(\rho)\right]=\left[\begin{array}{ccc}
V_{1}(\rho) & V_{2}(\rho) & 0 \\
i & i & 0 \\
0 & 0 & 1
\end{array}\right],
$$

where $V_{k}(\rho)=-i \sigma\left(\tau_{k}(\rho)\right)(F+L) /\left(C+L \sigma^{2}\left(\tau_{k}(\rho)\right)\right)$. Other constants such as $L_{i j \alpha}$ and $M_{\alpha p}$ which are of relevance to our computation here can be calculated directly using (6.5).

Firstly, consider the case where the cracks are subject to an antiplane deformation, i.e. we take

$$
P_{1}=0, P_{2}=0 \text { and } P_{3}=s_{0}(\sin \theta+\cos \theta),
$$

where $s_{0}$ is a real positive constant.

For the antiplane case, the coefficients $\alpha_{1}^{n}$ and $\alpha_{2}^{n}$ are zero. We solve (4.20) for $\alpha_{3}^{n}$, and use (5.3) to compute the non-dimensionalised crack energy $L U /\left(s_{0} d\right)^{2}$. In our computation, we employ no more than 10 terms in the series approximation (4.19). For $R=2 L /(A-N)=1.334$ (titanium), $a / d=0.250$ and $h / d=2.500,3.500$ and 4.500 as well as for $h / d$ tending to infinity, we plot $L U /\left(s_{0} d\right)^{2}$ against $\theta$ in Figure 2. From Figure 2, it is obvious that, for a fixed value of $\theta$, the crack energy increases as $h / d$ decreases. Also, note that, for a given value of $h / d$, the crack energy is a maximum when $\theta=\theta_{0}$, where $0<\theta_{0}<\pi / 2$. For the case where $h / d$ tends to infinity, $\theta_{0}=\pi / 4$. For the cases considered here, it is apparent 


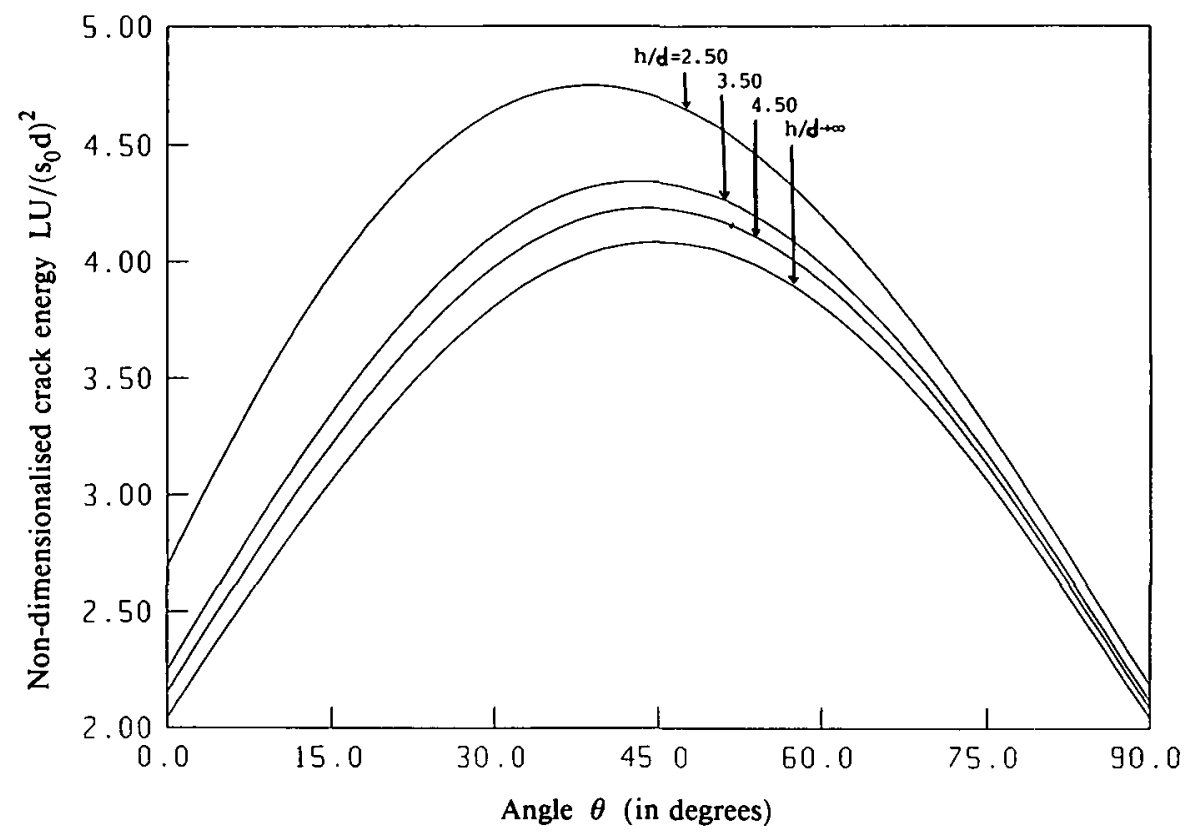

FIGURE 2. Variations of $L U /\left(s_{0} d\right)^{2}$ with $\theta$ for $a / d=0.25, \quad R=1.334$ (titanium) $h / d=2.50,3.50$ and 4.50 as well as $h / d$ tending to infinity.

that decreasing the value of $h / d$ has the effect of decreasing $\theta_{0}$. For $a / d=$ $0.500, h / d=5.000$ and $R=0.500,1.000$ (isotropic case) and 2.000, the non-dimensionalised crack energy is plotted against $\theta$ in Figure 3. It is clear from Figure 3 that the crack energy for $R=1.000$ is less or greater than that for $R=2.000$ or $R=0.500$ respectively.

We now consider the case where the cracks are opened up by constant tensile tractions acting on their faces. Specifically, we choose $P_{k}(x)$ as

$$
P_{1}=T_{0} \cos \theta, \quad P_{2}=T_{0} \sin \theta \text { and } P_{3}=0,
$$

where $T_{0}$ is a given positive constant.

To obtain some numerical results, we use the elastic constants for titanium. These constants are given by $A / L=3.469, N / L=1.970, C / L=3.876$ and $F / L=1.478$. Using these constants, we solve (4.20) and employ (5.3) to calculate the non-dimensionalised crack energy $L U /\left(T_{0} d\right)^{2}$. The results for $a / d=0.250$ and selected values of $\theta$ and $h / d$ are given in Table 1 . From the table, it is obvious that for a given value of $\theta$, decreasing $h / d$ has the effect of increasing the crack energy. Note that for $h / d=4.500$ as well as for $h / d$ tending to infinity, the crack energy is an increasing function of $\theta$ while for $h / d=2.500$ it is a decreasing function of $\theta$. In Table 


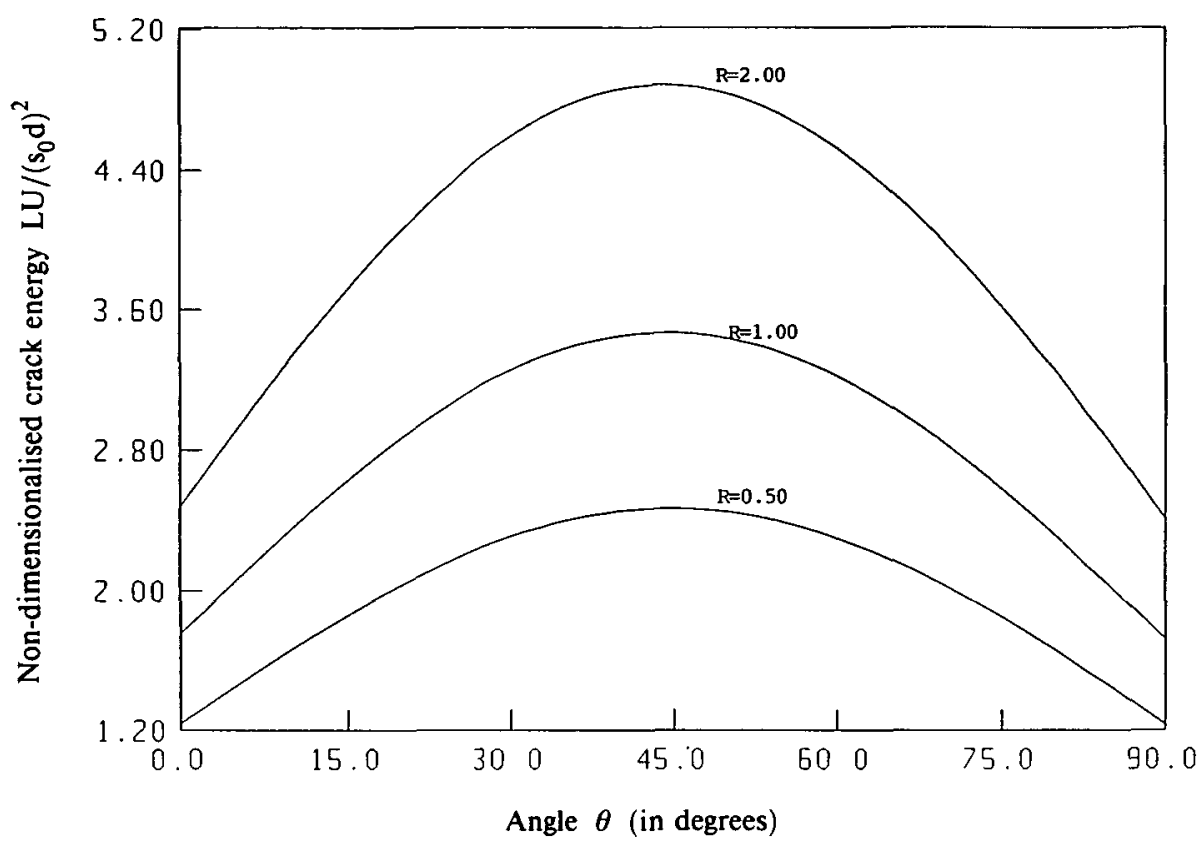

FIGURE 3. Variations of $L U /\left(s_{0} d\right)^{2}$ with $\theta$ for $a / d=0.25, R=1.334$ (titanium) and $h / d=2.50,3.50$ and 4.50 as well as $h / d$ tending to infinity.

2 , for $\theta=\pi / 3$ and $h / d=3 / 2$, we examine the effect of changing $a / d$ $(0<a / d<1)$ on the crack energy $L U /\left(T_{0} d\right)^{2}$. There is a critical value of $a / d$ for which the crack energy is minimum, and as $a / d$ tends to 0 (from above) or 1 (from below) the crack energy tends to infinity.

TABLE 1. Non-dimensionalised crack energy for $a / d=0.250$ and selected values of $\theta$ and $h / d$ (plane problem involving a titanium slab).

\begin{tabular}{|c|c|c|c|c|}
\hline \multicolumn{5}{|c|}{$L U /\left(T_{0} d\right)^{2}$} \\
\hline $\begin{array}{c}h / d \\
\theta\end{array}$ & 2.500 & 3.500 & 4.500 & $h / d \rightarrow \infty$ \\
\hline $0^{\circ}$ & 1.656 & 1.316 & 1.243 & 1.157 \\
\hline $30^{\circ}$ & 1.584 & 1.358 & 1.284 & 1.173 \\
\hline $60^{\circ}$ & 1.527 & 1.398 & 1.333 & 1.206 \\
\hline $90^{\circ}$ & 1.474 & 1.392 & 1.341 & 1.223 \\
\hline
\end{tabular}


TABLE 2. Non-dimensionalised crack energy for $\theta=\pi / 3, h / d=3 / 2$ and selected values of $a / d$ (plane problem involving a titanium slab).

\begin{tabular}{|c|c|c|c|c|c|c|c|c|c|}
\hline$a / d$ & 0.100 & 0.200 & 0.300 & 0.400 & 0.500 & 0.600 & 0.700 & 0.800 & 0.900 \\
\hline$L U /\left(T_{0} d\right)^{2}$ & 2.300 & 2.117 & 2.090 & 2.150 & 2.286 & 2.508 & 2.823 & 3.224 & 3.692 \\
\hline
\end{tabular}

\section{Acknowledgements}

Part of the research for this paper was carried out in the Department of Mathematics at Iowa State University. I wish to thank Mr. K. H. Lau of the Department of Chemical Engineering at Iowa State University for helping me to obtain some of the numerical results presented and Dr. M. Naim Yunus, a helpful and supportive colleague for introducing me to the various facilities at Universiti Sains Malaysia.

\section{References}

[1] M. Abramowitz and I. A. Stegun (eds), Handbook of Mathematical Functions (Dover, New York, 1970).

[2] W. T. Ang, "A cracked anisotropic elastic slab", Int. J. Engng. Sc. 26 (1988) 277-283.

[3] W. T. Ang, "An arbitrarily-oriented plane crack in an anisotropic elastic slab", Engng. Fract. Mech. 32 (1989) 965-972.

[4] W. T. Ang, "Transient response of a crack in an anisotropic strip", Acta Mechanica 70 (1987) 97-109.

[5] D. L. Clements, “A crack in an anisotropic elastic slab”, Quart. Appl. Maths 34 (1977) 437-443.

[6] D. L. Clements and T. R. Tauchert, "Deformation of an anisotropic slab containing a crack", Acta Mechanica 32 (1979) 55-61.

[7] R. S. Dhaliwal, "Two coplanar cracks in an infinitely long orthotropic slab", Utilitas Mathematica 4 (1973) 115-128.

[8] I. S. Gradshtein and I. M. Ryzhik, Table of Integrals, Series and Products (Academic Press, San Diego, 1980).

[9] D. L. Hill and D. L. Clements, "On deformations of cracked anisotropic slabs", J. Elast. 14 (1984) 403-413.

[10] N. I. Loakimidis, "A new singular integral equation for the classical crack problem in plane and antiplane elasticity", Int. J. Fract. 21 (1983) 115-122.

[11] A. C. Kaya and F. Erdogan, "On the solution of integral equations with strongly singular kernels", Quart. Appl. Math. 45 (1987) 105-122.

[12] Y. Konishi, "Two coplanar cracks in an infinite transversely isotropic medium", Int. $J$. Engng. Sc. 10 (1972) 917-923.

[13] S. Krenk, "The stress distribution in an infinite plate with co-linear cracks", Int. J. Solids Structures 11 (1975) 449-460.

[14] I. N. Sneddon, Fourier Transforms (McGraw-Hill, New York, 1951).

[15] A. J. M. Spencer, Deformations of fibre-reinforced materials (Oxford University Press, 1972).

[16] A. N. Stroh, "Dislocations and cracks in anisotropic elasticity", Phil. Mag. 3 (1958) 625-646. 